\title{
Research Paper \\ Prediction of Executive Functions Based on Impairment in Motor and Linguistic Growth
}

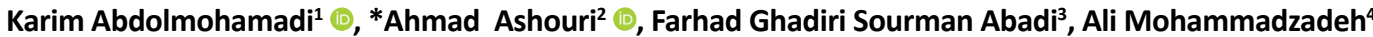

1. Department of Psychology, Faculty of Psychology \& Educational Sciences, Allameh Tabataba'i University, Tehran, Iran.

2. Department of Clinical Psychology, School of Behavioral Sciences and Mental Health (Tehran Institute of Psychiatry), Iran University of Medical Sciences, Tehran, Iran. 3. Department of Psychology, Faculty of Literature and Humanities, Urmia University, Urmia, Iran.

4. Department of Psychology, Payame Noor University, Tehran, Iran.

\begin{tabular}{|c|c|}
\hline $\begin{array}{l}\text { Use vour devicict to scan } \\
\text { and read the article online }\end{array}$ & dttation Abdolmohamadi k, Ashouri A, Ghadiri Sourman Abadi F, Mohammadzadeh A. [Prediction of Executive Functions \\
\hline aristing & $\begin{array}{l}\text { Based on Impairment in Motor and Linguistic Growth (Persian)]. Iranian Journal of Psychiatry and Clinical Psychology. 2020; } \\
\text { 26(2):188-199. http://dx.doi.org/10.32598/ijpcp.26.2.227.12 }\end{array}$ \\
\hline 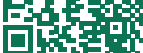 & doli'http://dx.doi.org/10.32598/ijpcp.26.2.227.12 \\
\hline
\end{tabular}

Received: 26 Jun 2019

Accepted: 16 May 2020

Available Online: $01 \mathrm{Jul} 2020$

Key words:

Growth, Movements Linguistic, Executive functions

\begin{abstract}
Objectives Executive functions refer to the use of cognitive processes to control thoughts and emotions. The purpose of this study was to predict impairment in executive functions, based on impairment in motor and linguistic growth in children.

Methods The research method is descriptive correlational. The statistical population of this study was all students of elementary school studying in the academic year of 2018-2019 in Tabriz City, AMONG them 700 were selected in a multi-stage cluster. In the next step, their parents were asked to complete the Coolidge Neuropsychological and Personality Questionnaire (2002) and FTF-5 -15 questionnaire. The data were analyzed by Simultaneous Regression tests.

Results The results showed that defective motor skills, language proficiency, and communication skills cause an executive function disorder.

Conclusion Language skills and motor development are factors that are effective in the growth of executive functions.
\end{abstract}

\section{Extended Abstract}

\section{Introduction}

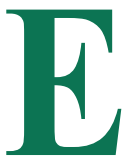

xecutive functions refer to the ability to use cognitive processes to control thoughts and emotions and this ability causes goaldirected behavior, therefore the executive functions necessary to succeed in the daily lives of all human beings. In neurobiological studies, it has been determined that the prefrontal cortex and its connections have a major impact on the growth of executive functions. The growth of the prefrontal cortex begins at birth and continues until puberty and this long-term growth is more influenced by environmental stimuli.

Research has shown that the main growth of executive functions occurs between the ages of 3 and 7 and continues gradually until the teenage years. Children in different periods are susceptible to the impact of their environment There is also research evidence that in early childhood, children in terms of the growth of executive functions, are more influenced by their environment. The research has shown that motor growth is one of the environmental factors affecting the growth of executive functions.

* Corresponding Author:

Ahmad Ashouri, PhD.

Address: Department of Clinical Psychology, School of Behavioral Sciences and Mental Health (Tehran Institute of Psychiatry), IranUniversity of Medical Sciences, Tehran, Iran.

Tel: +98 (21) 66551656

E-mail: ahmad.ashouri@gmail.com 
Motor growth refers to progressive changes in motor behavior throughout life, which is the result of the interaction between task needs, individual biological characteristics, and environmental conditions. Studies conducted by researchers who examined the effects of movements on executive functions in adolescents showed that children's executive functions are influenced by their motor growth. The other components of the environment associated with executive functions are language development. In this regard, the research has identified, children with language impairments also have weaknesses in executive functions. Given the existing vacuum and the lack of coherent research that examines the impact of environmental factors such as motor and lingual skills growth on executive functions, the present study was conducted with the purpose prediction of impairment in executive functions based on defects in motor and linguistic growth.

\section{Methods}

The research method is descriptive correlational. The statistical population of this study was all students of elementary school studying in the academic year of 2018-2019 in Tabriz City that 700 of them were selected through multistage cluster sampling. In the next step, their parents were asked to complete The Coolidge Personality and Neuropsychological Questionnaire (2002) and FTF-5 -15 Growth Inventory.

\section{Results}

The findings of this study showed that the effect of the defect in the received language (0.19) and defect in the communication language $(0.25)$ on the executive function disorder was significant at the level of 0.0001 . Also, the effect of the defect in large movements (0.12) is significant on the incidence of executive functions at the level of 0.004; however, defects in expressive language and delicate movements do not have a significant effect on the prediction of executive function disorder. Hence, the lack of large motor skills, receptive language skills, and communication language skills can lead to executive function disorder.

\section{Discussion}

The present study showed that defect in the receptive and communication language is effective in predicting executive function disorder. This finding is consistent with the results of some studies. In explaining the relationship between language deficits and disruptions in executive functions, the function of brain structures, especially the prefrontal lobe, can be used.
Given that language development facilitates the transfer of people's experiences, helps people without direct experience, and uses concepts to intervene in various affairs that will result in the growth of higherlevel cognitive processes such as executive functions.

Of the etiology of the relationship between deficits in motor skills and executive function disorder, it can be argued that physical activity by controlling the maintenance, growth, and differentiation of neurons, synapses, and angiogenesis improves neurotrophy and, as a result, improves cognitive functions such as processing speed, control strategies, and scheduling and working memory.

Another explanation for the relationship between motor growth and executive functions can be raised. Given that the three main executive functions strategies - inhibition, working memory, and shifting attention - are affected by the development of the cerebellum, whose development is also influenced by the motor growth, especially the delicate movements. The development of motor skills plays a role in the growth of executive functions by facilitating the development of the cerebellum. Language skills and motor development are factors effective in improvement of executive functions in children.

\section{Ethical Considerations}

\section{Compliance with ethical guidelines}

All participants were informed about the study Objectives and its potential benefits and they were assured of the confidentiality of their information. They were free to leave the study at any time.

\section{Funding}

This research did not receive any specific grant from funding agencies in the public, commercial, or not for profit organizations.

\section{Authors contributions}

Investigation and draft preparation: Karim Abdolmohamadi; Project administration, supervision, editing \& review: Ahmad Ashouri; Resource and data analysis: Farhad Ghadiri Sourman Abadi; methodology, editing \& review: Ali Mohammadzadeh. 


\section{Conflicts of interest}

The authors declare no conflict of interest

\section{Acknowledgements}

The authors would like to thank the head and staff of Education Departments in five districts of Tabriz city, and the participants for their valuable cooperation. 


\title{
يبشبينى اختلال در كاركردهاى اجرايى بر اساس نقص در رشد حركتى وزبانى
}

\author{
كريم عبدالمحمدى :)، "احمد عاشورى [i)، فرهاد غديرى صورمان آبادى، على محمدزاده

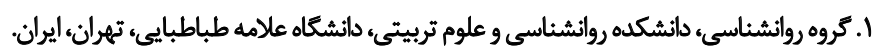

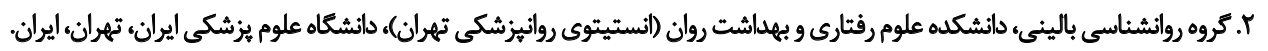

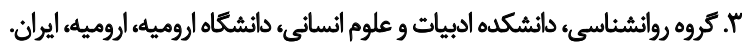

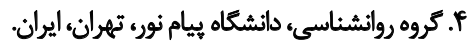

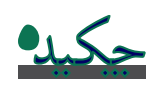

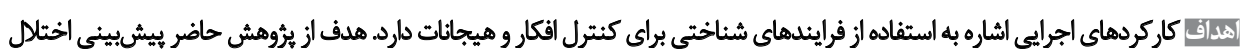

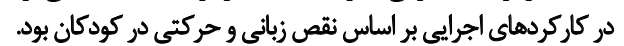

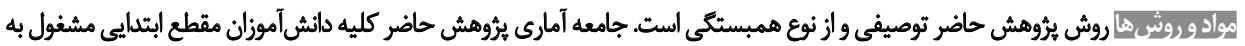

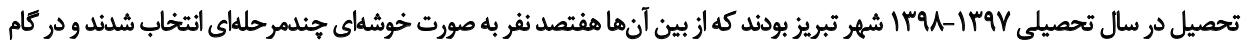

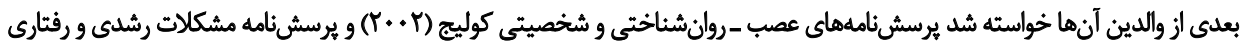

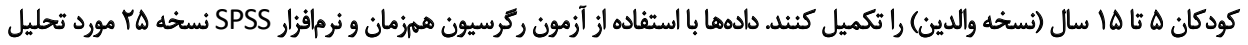
قراركزفتنند.

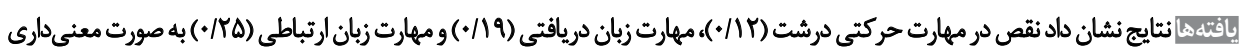

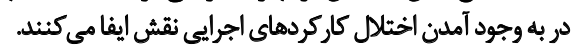

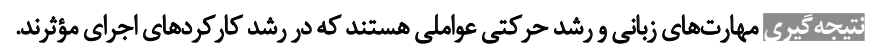

دانست كه در زندگى و انجام تكاليف يادگيرى و كنش هاى هوشى

data

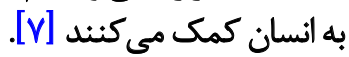

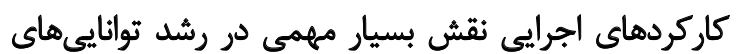

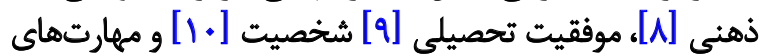

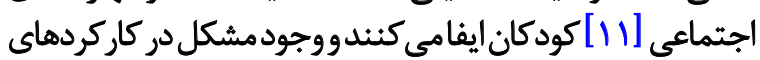

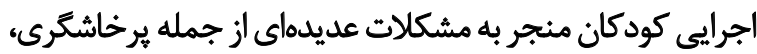

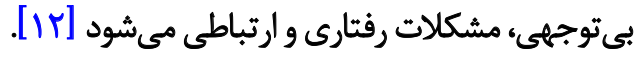

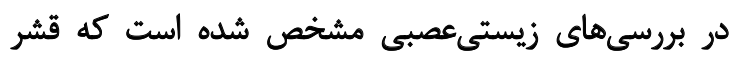

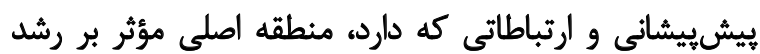

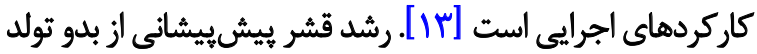

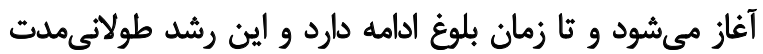

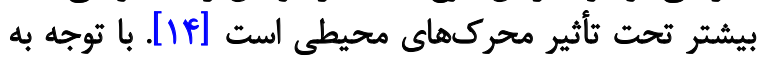

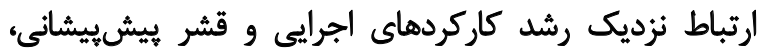

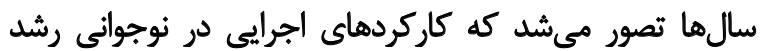

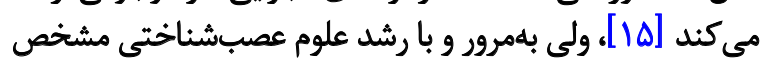

امروزه اين ديدكاه كه رشد مغز افراد تحت تأثير محيط اوليه

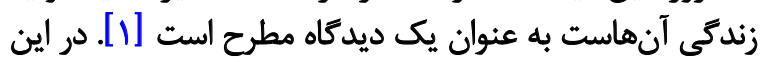

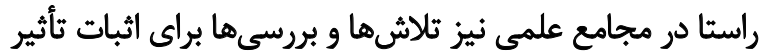

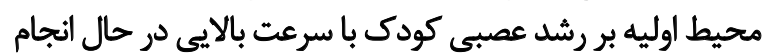

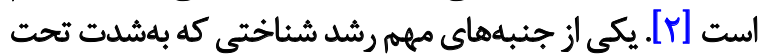

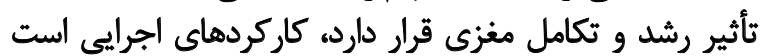

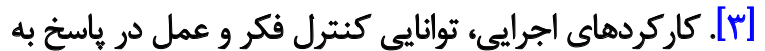

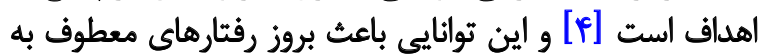

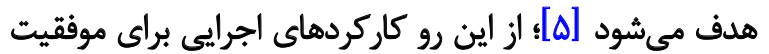

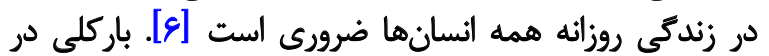

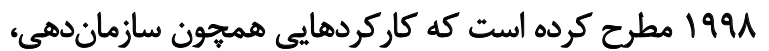

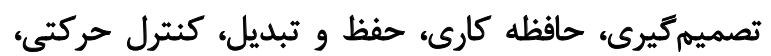

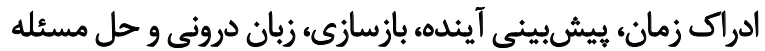

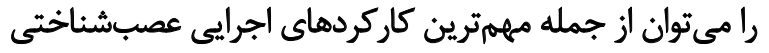


كودكان با اختلال زبان بيانى، در بيان عواطف، احساسات، عقايد

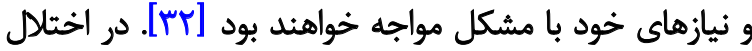

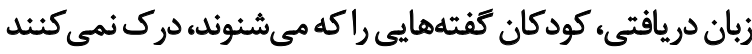

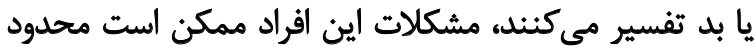

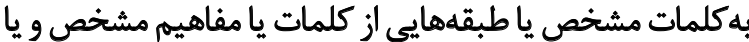
ساختارهاى دستورى باشد [اسب].

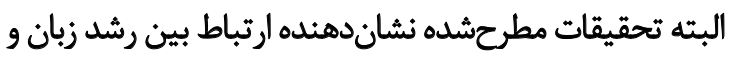

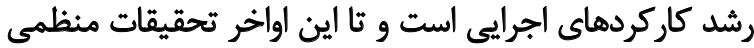

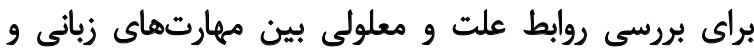

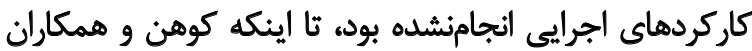

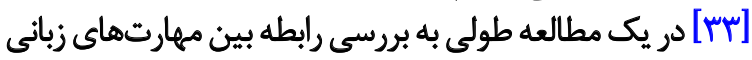

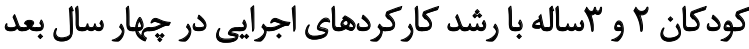

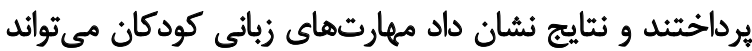

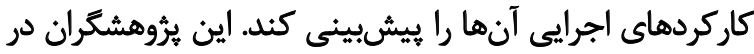

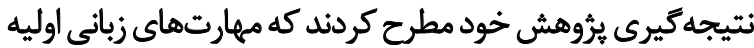

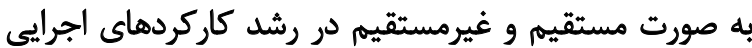

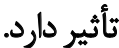

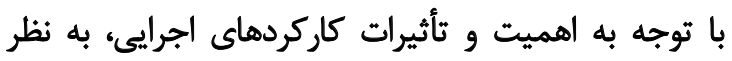

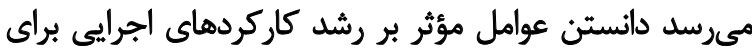

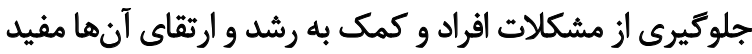

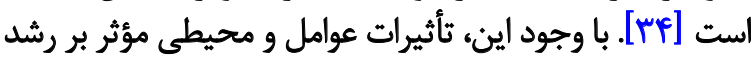

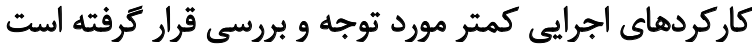

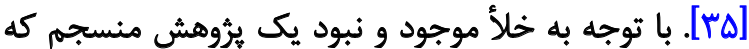

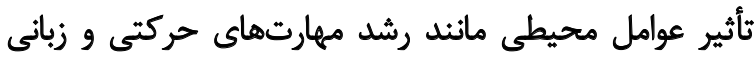

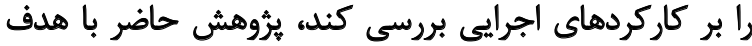

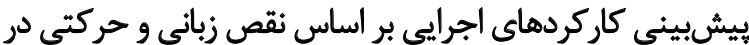

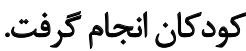

روش ئزوهش حاضر توصيفى و از نوع همبستئى است. جامعه

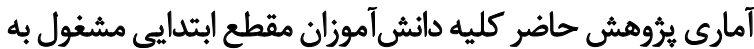

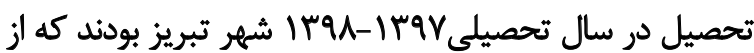

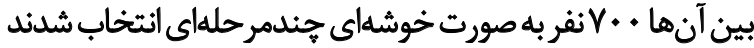

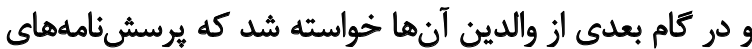

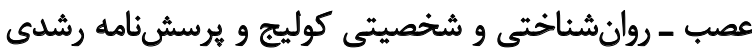

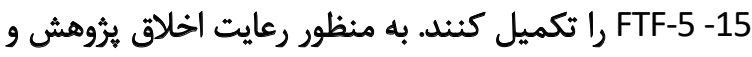

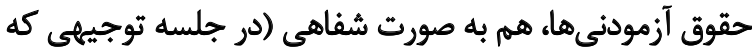

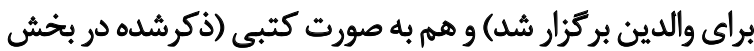

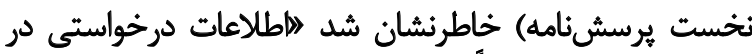

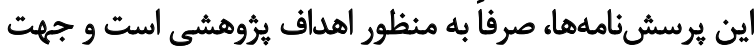

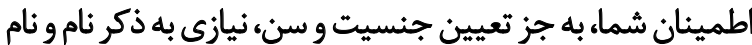

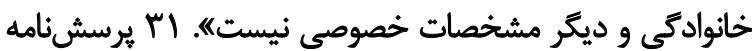

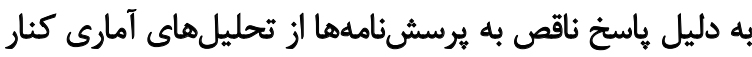

شد كه كاركردهاي اجرايى بسيار زودتر و قبل از دوره دبستان

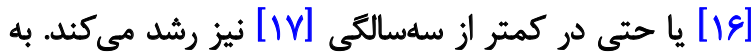

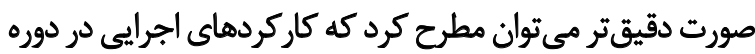
نوزادى شكل مى

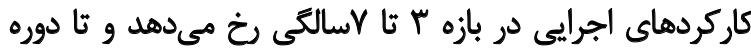

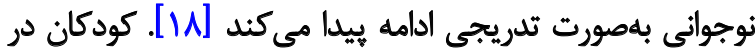

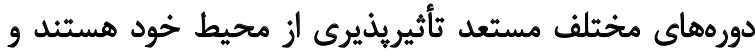

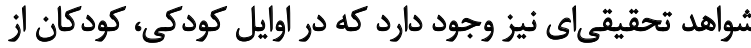

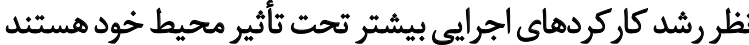

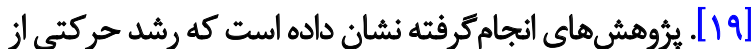

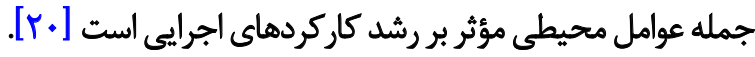
رشد حركتى به تغييرات بيش رورنده در رفتار حركتى در سراسر

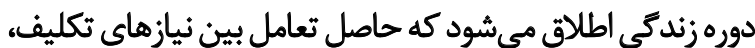

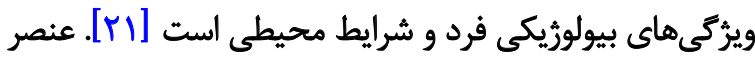

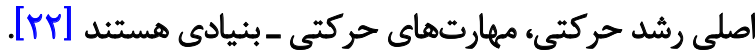

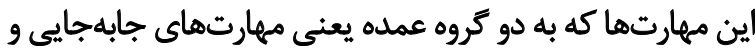

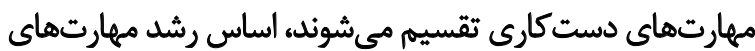

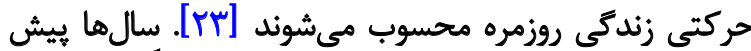

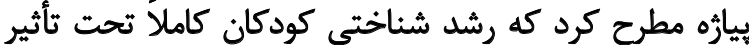

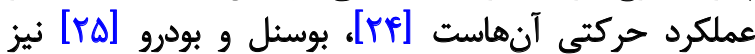

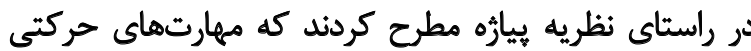

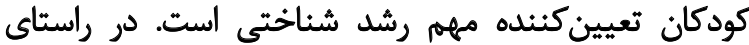

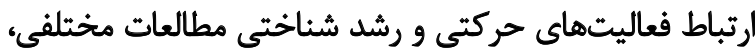

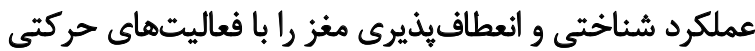

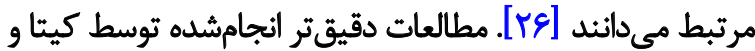

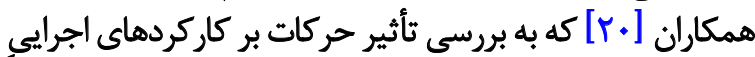

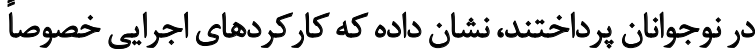
سطح توجه و حافظه كودكان تحت ثأثير رشد حركتئ ناركان آنهاست

از ديكر مؤلفههايى كه به نظر مىرسد با كاركردهاى اجرايى

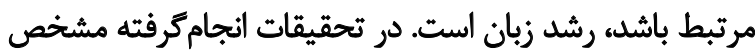

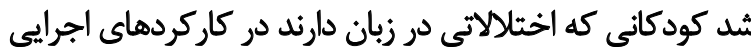

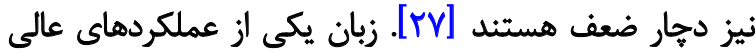

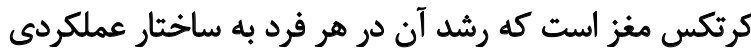

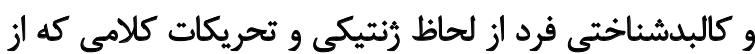

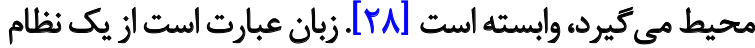

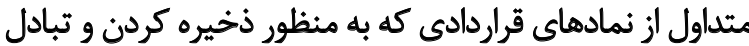

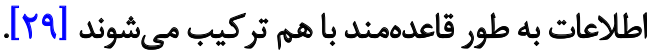
نقص در زبان، در اثر وارد آمدن ضايعه مغزى و اختلال در

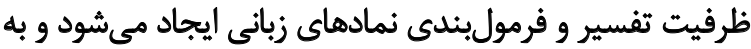

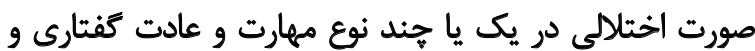

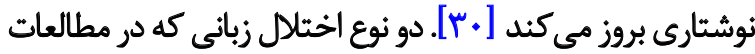

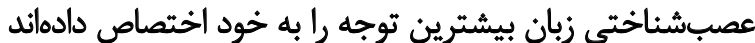

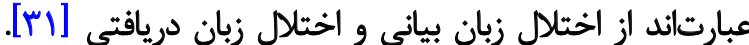


دو زيرمؤلفه نقص در رشد مهارتهاى حركتي و زبانى به ترتيب

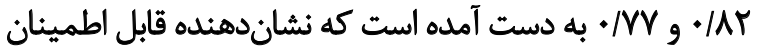

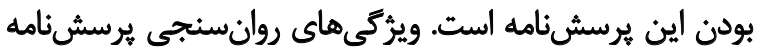

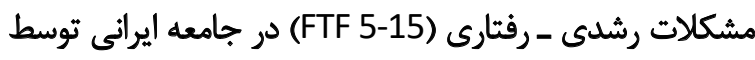

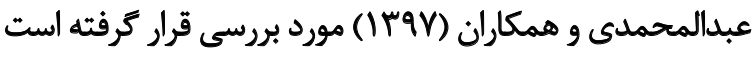

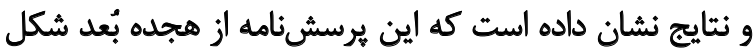

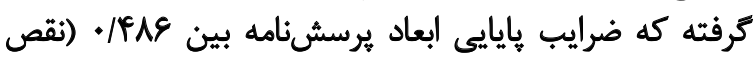

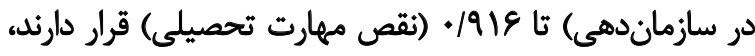

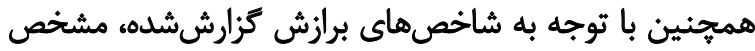

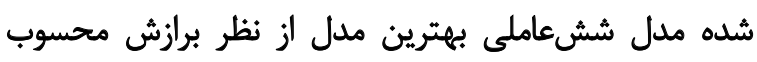

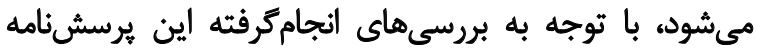

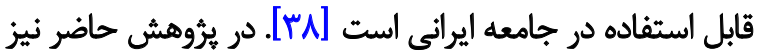

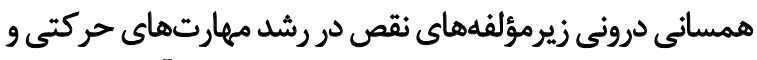

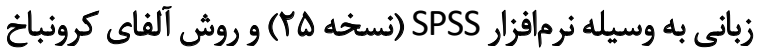

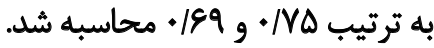

يافتهاها

قبل از بررسى سهم متغيرهاى بيشبين كاركردهاى اجرايى

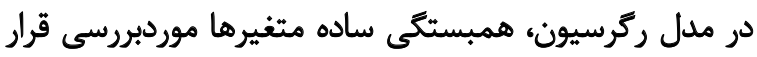

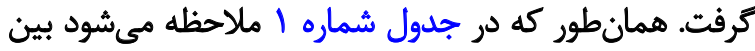

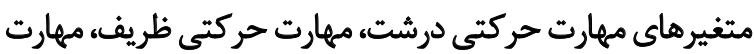

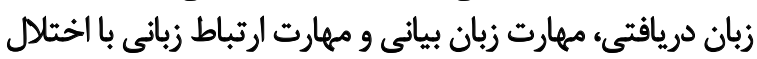
در كاركردهاى اجرايى همبستكى وجود داردي.

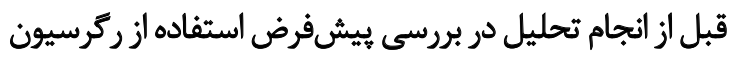

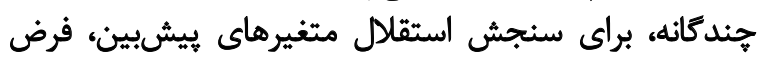

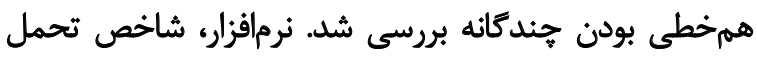

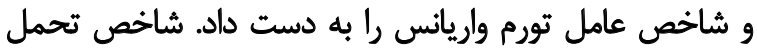

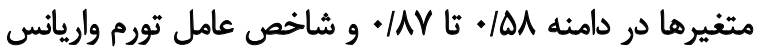

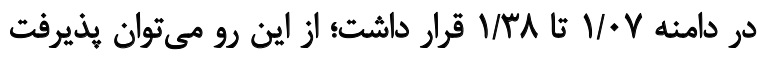

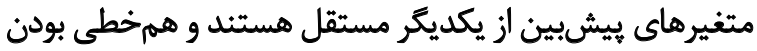

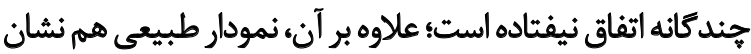

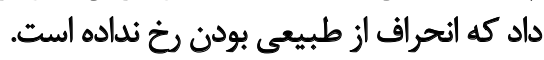

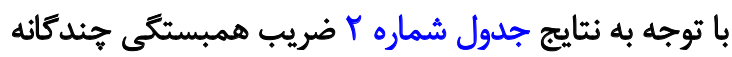

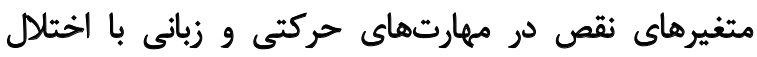

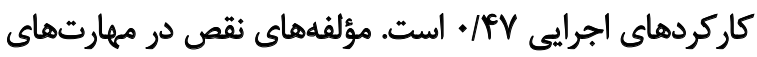

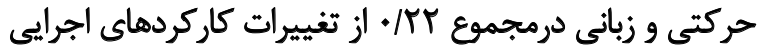

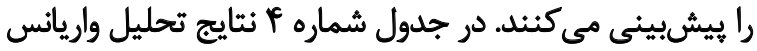

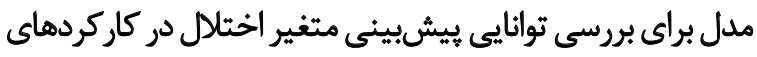

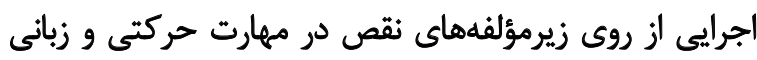

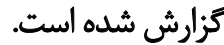

در جدول شماره ب خلاصه نتايج ركرسيون كزارش شده

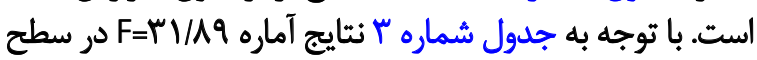

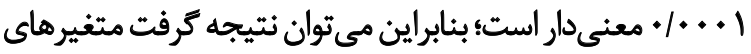

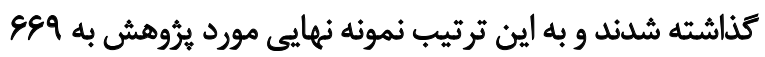

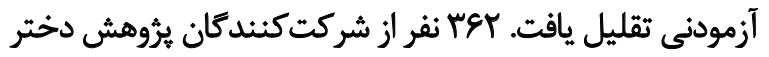
با ميانكين سنى

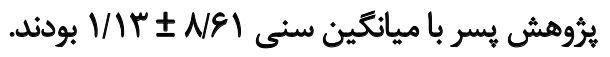

\section{أبزار تحقيق}

\section{هرسش نامه عصب -روانشناختى و شخصيتى كوليج'}

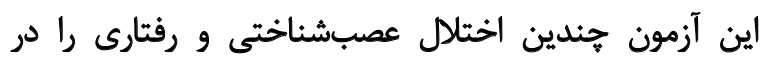

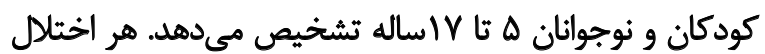

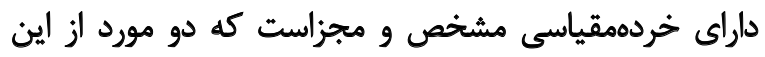

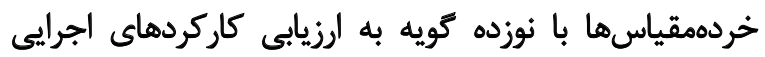

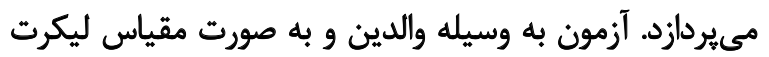

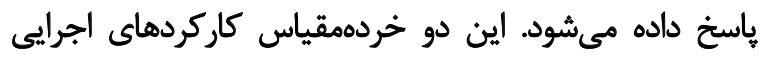

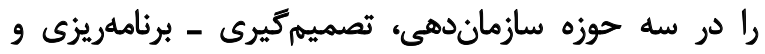

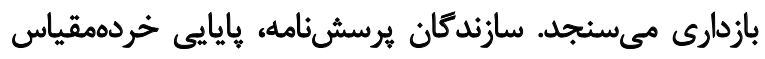

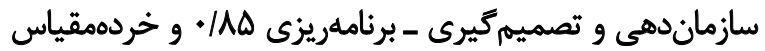

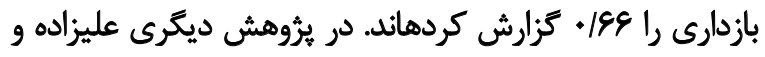

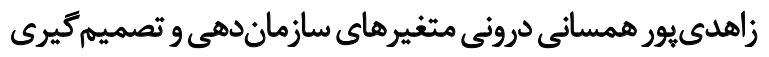

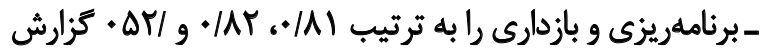

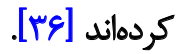

يرسش (نسامه مشكلات رشدى و رفتارى كودكان هـ تا هالدين سال (نسخه والدين) اين برسشنامه داراى ||l| آيتم است كه به صورت ليكرت

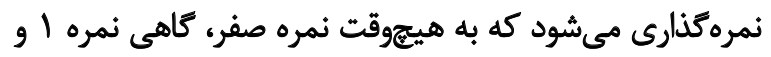

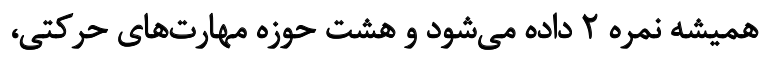

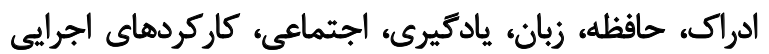

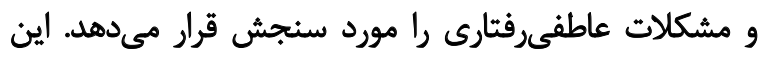

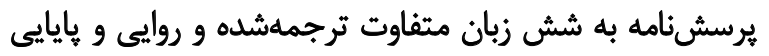

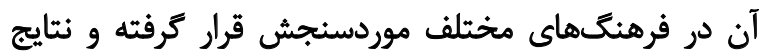

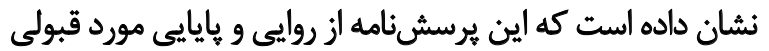

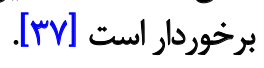
در يُروهش حاضر از قسمت بررسى نقص در مهارتهاى زبانى زئى

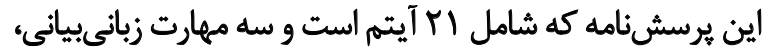

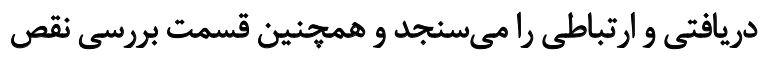

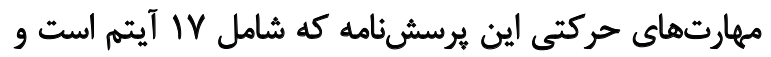

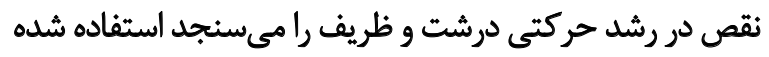

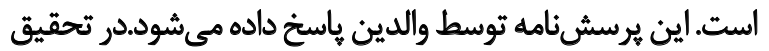

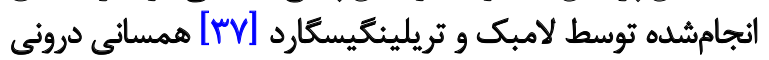

1. Coolidge Neuropsychological And Personality Scale

2. Parent questionnaire for evaluation of development and behaviour in 5-15 Year old children (FTF-5 -15) 
جدول ا. همبستكى ساده بين اختلال كاركردهاى اجرايى با نقص در مهارتهاى حركتى و زبانى

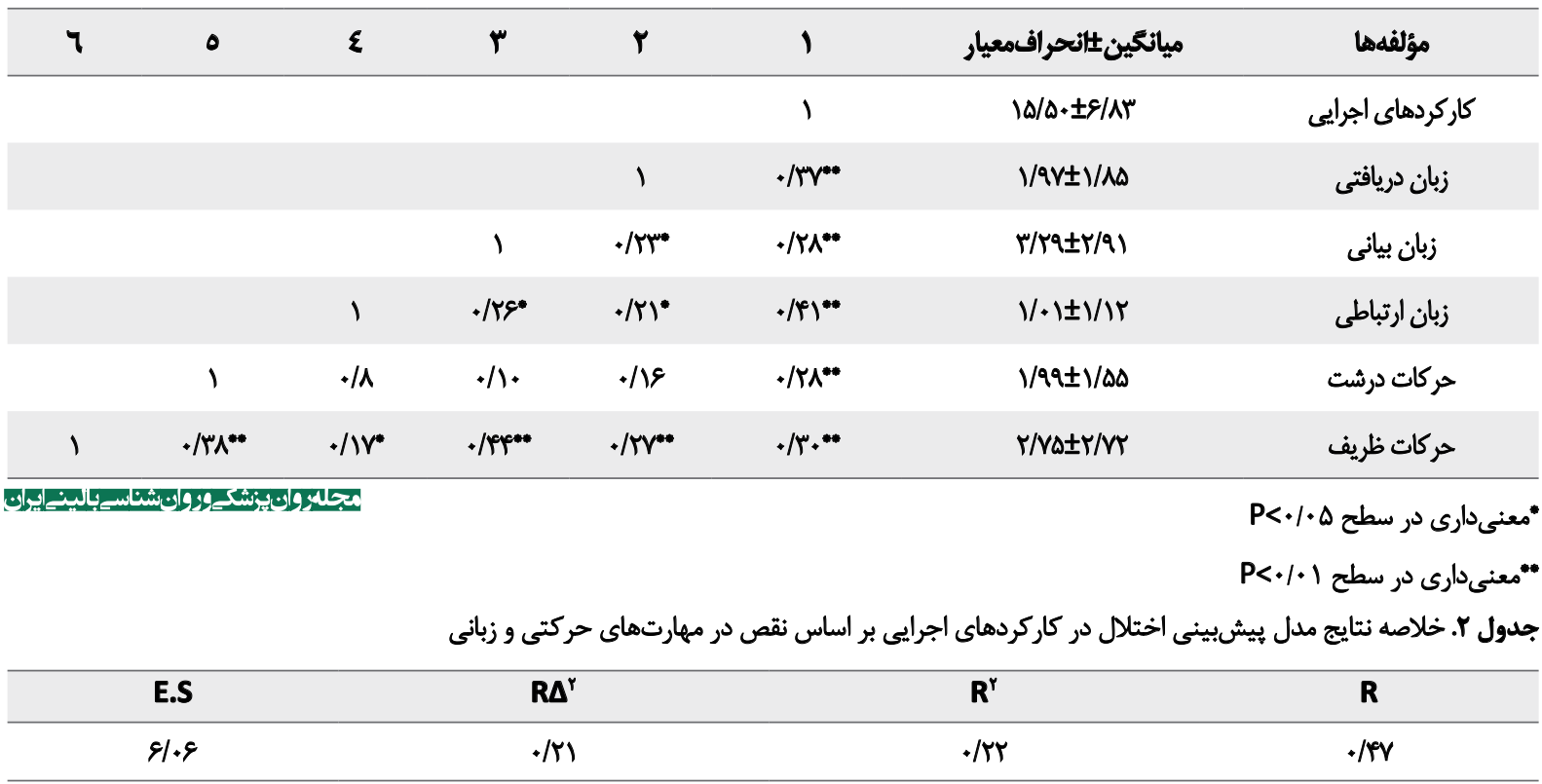

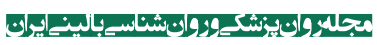

جدول ب. نتايج تحليل واريائس ركرسيون جندمتفيرى متغير بيشبين از روى متغير ملاك

\begin{tabular}{|c|c|c|c|c|c|}
\hline Sig & $\mathbf{F}$ & MS & df & SS & مدل \\
\hline \multirow[t]{3}{*}{$.1 . . .1$} & $\mathrm{rV} / \mathrm{Aq}$ & $I I V \in / \Delta T$ & $\Delta$ & $\Delta A Y T / G T$ & ركرسيون \\
\hline & & re/AT & $\Delta T^{\prime}$ & $r \cdot \Delta \varphi g / \Delta \theta$ & باقىمانده \\
\hline & & & $\Delta 81$ & rePIV/AA & كل \\
\hline
\end{tabular}

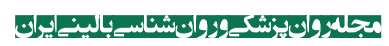

بيشبين (نقص در زبان دريافتى، زبان بيانى، زبان ارتباطى، ضرايب استانداردشده و استانداردنشده ركرسيون كزارششدهاند.

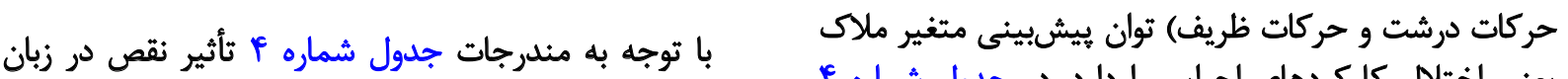

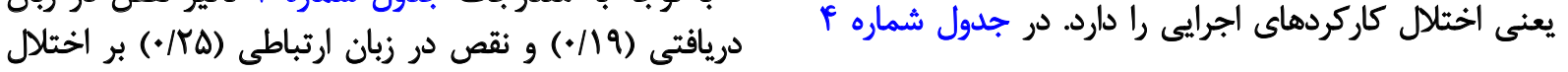

جدول f. ضرايب ركرسيون استاندارد نشده و استانداردشده مدل بيشبينى اختال كاركردهاى اجرايى

\begin{tabular}{|c|c|c|c|c|c|}
\hline \multirow{2}{*}{$\mathbf{P}$} & \multirow{2}{*}{$\mathbf{T}$} & \multirow{2}{*}{ ضرايب استائداردثشده } & \multicolumn{2}{|c|}{ ضرايب استاندار دنشده } & \multirow{2}{*}{ مدل } \\
\hline & & & SE & B & \\
\hline $.1 . . .1$ & $r e f . r$ & & .178 & $11 / 18$ & علد ثابت \\
\hline $.1 . . .1$ & m/qr &.$/ 19$ &.$/ 18$ &.$/ N$ & زبان دريافتى \\
\hline.$N E$ &.$/ 7 q$ &.$/ .1$ &.$/ 11$ &.$/ m$ & زبان يياني \\
\hline$+1+.+1$ & $\Delta / \pi$ &.$/ T \Delta$ & $\cdot / \mathrm{KA}$ & V/AP & زبان ارتباطى \\
\hline 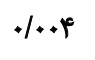 & r/Aq &.$/ 1 T$ &.$/ 19$ & $.1 \Delta F$ & حركات درشت \\
\hline r &.$/ p r$ &.$/ . r$ &.$/ 11$ &.$/ \Delta E$ & حركات ظريف \\
\hline
\end{tabular}




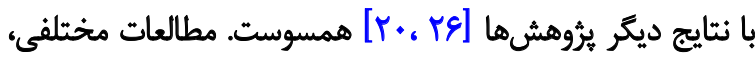

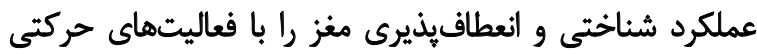

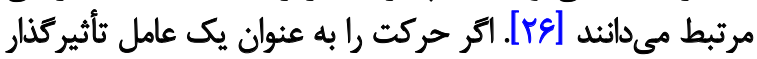

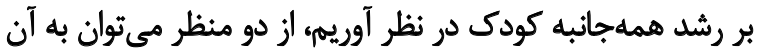

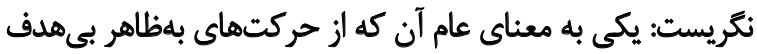

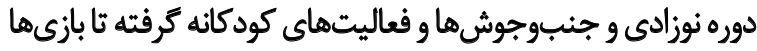

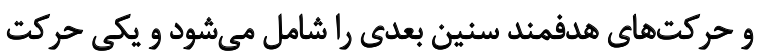

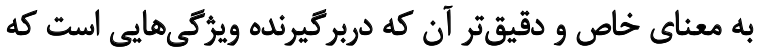

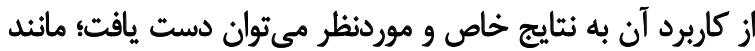

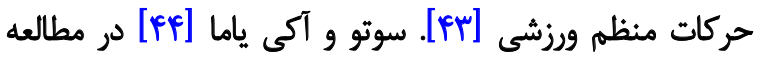

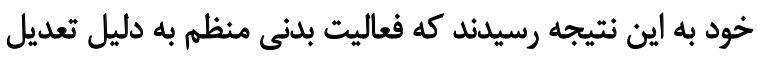

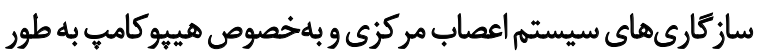

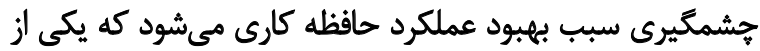

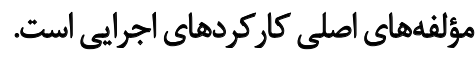

درباره سببشناسى اين ارتباط مي توان هنين مطرح كرد كه

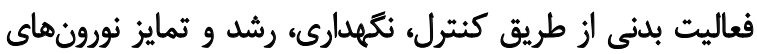

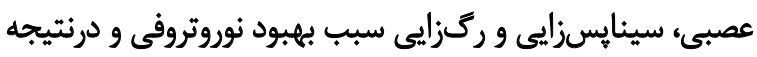

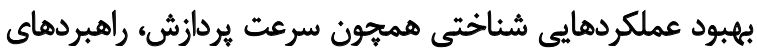

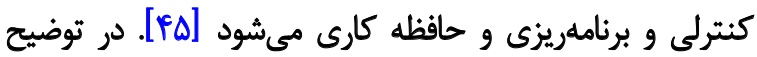

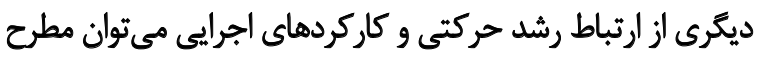

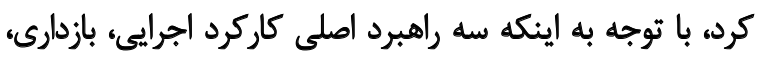

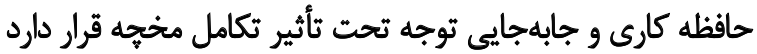

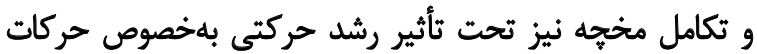

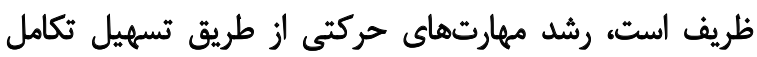

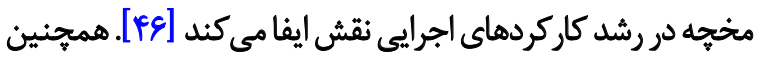

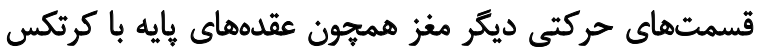

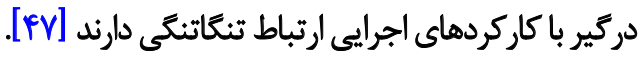

\section{تئيجإكيرى}

مجموعاً با توجه بيشينه يروهش و نتايج تحقيق حاضر، به نظر

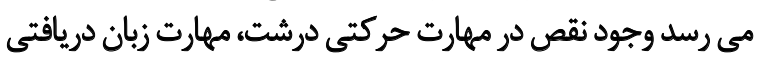

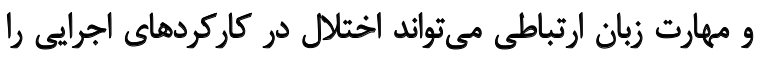

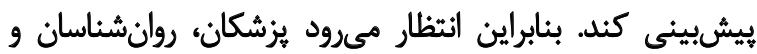

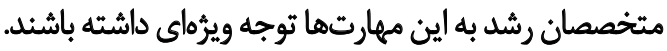

يثروهش حاضر از لحاظ جامعه آمارى داراى محدوديت بوده و به يك

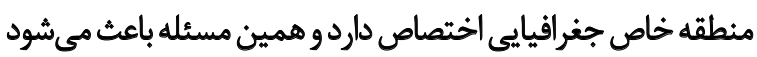

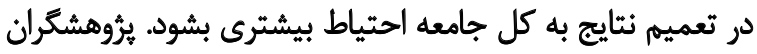

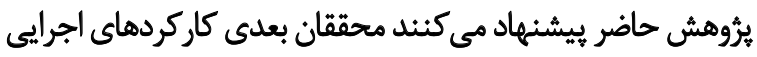

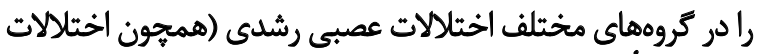

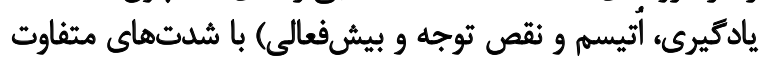

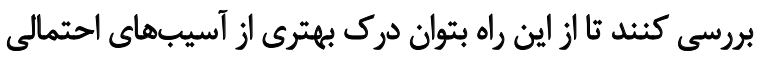
در اين زمينه به دست آلورد.

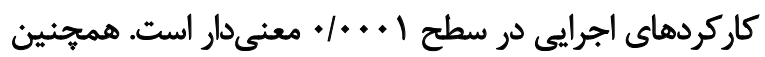

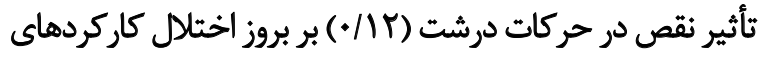

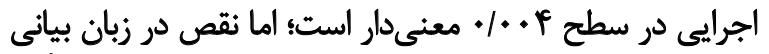

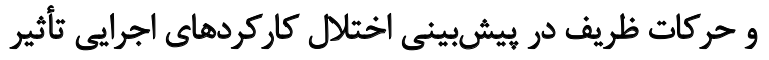

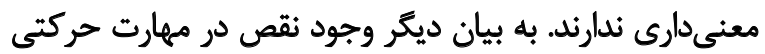

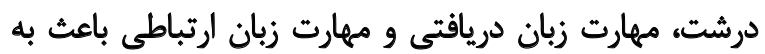

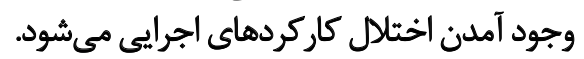

ب

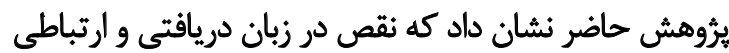

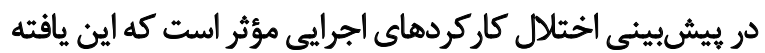

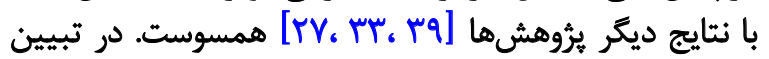

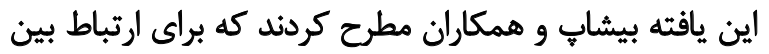

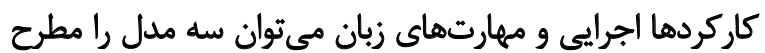

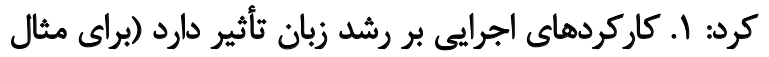

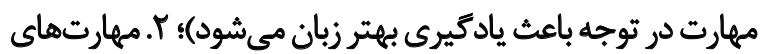

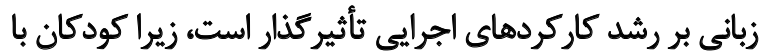

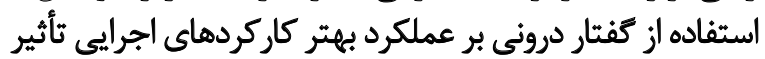

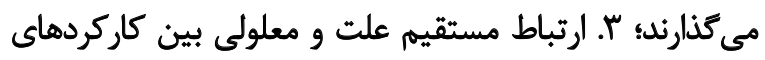

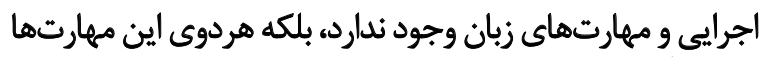

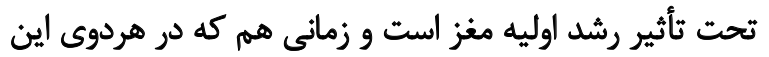

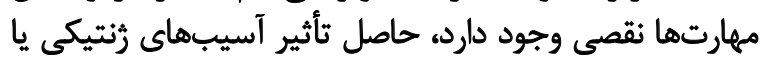

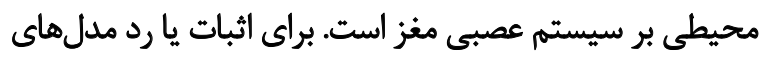

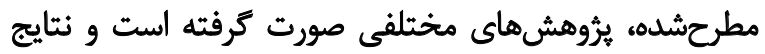

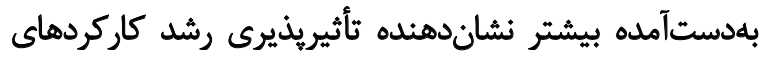

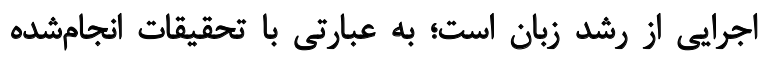

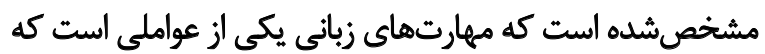

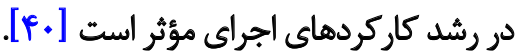

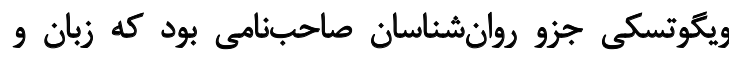

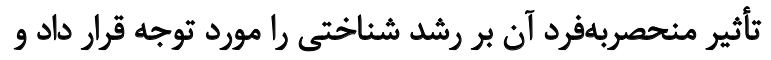

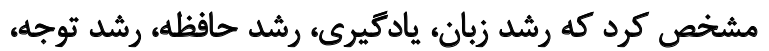

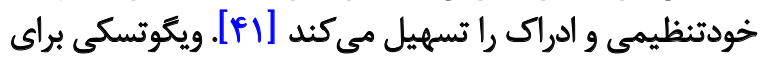

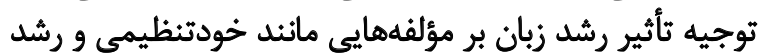

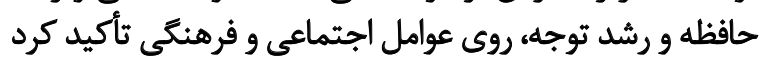

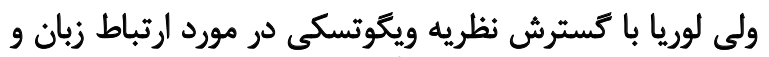

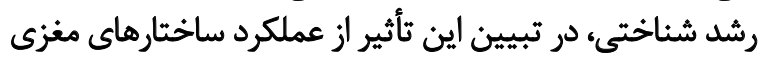

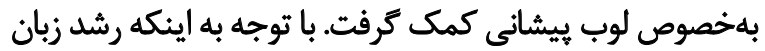

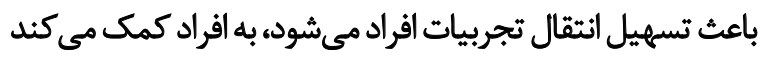

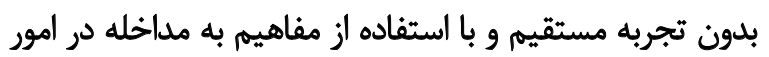

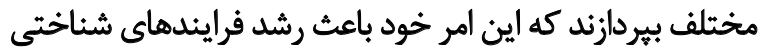

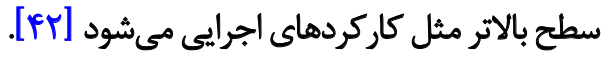

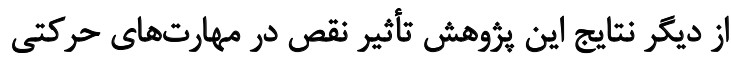

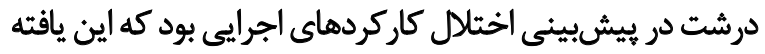




\section{ملاحظات اخلاقى}

$$
\text { بيروى از اصول اغلاق ثئوهش }
$$

همه شركت كنند أنان در مورد اهداف مطالعه و مزاياى بالقوه

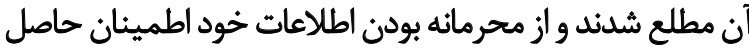

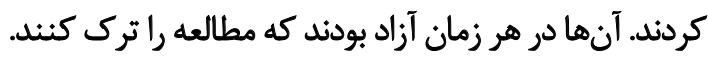

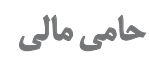

هيروهش حاضر هيجَّونه حمايت مالى دريافت نكرده است.

$$
\text { مشار كت نويسند مَان }
$$

تحقيق و بررسى، نكارش بيشنويس: كريم عبدالمحمدى؛

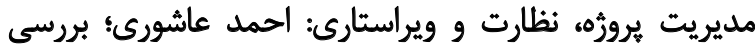

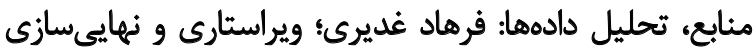
نوشته، روش شناسى: على محمدزاده.

$$
\text { تعارض مثافع }
$$

بنابر اظهار نويسندكان يرؤهش حاضر هيجّونه تعارض منافعى

$$
\text { تشكر و قدردانى }
$$

از مديران و كاركنان مناطق جنجانانه آموزش و و يرورش

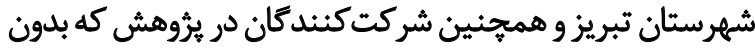

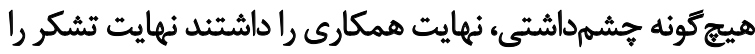




\section{References}

[1] Gunnar MR, Fisher PA. The Early Experience, Stress and Prevention Network. Bringing basic research on early experience and stress neurobiology to bear on preventive interventions for neglected and maltreated children. Development and Psychopathology. 2006; 18(3):651-77. [DOI:10.1017/S0954579406060330] [PMID]

[2] Nelson CA, Bloom FE. Child development and neuroscience. Child Development. 1997; 68(5):970-87. [DOI:10.2307/1132045] [PMID]

[3] Garon N, Bryson SE, Smith IM. Executive function in preschoolers: A review using an integrative framework. Psychological Bulletin. 2008; 134(1):31-60. [DOI:10.1037/0033-2909.134.1.31] [PMID]

[4] Lariche Z, Haghayegh SA. [The comparison of executive functions, risk behaviors, and academic motivation among adolescents with chronology type morningness and eveningness (Persian)]. Iranian Journal of Psychiatry and Clinical Psychology. 2018; 23(4):438-53. [DOI:10.29252/nirp.ijpcp.23.4.438]

[5] Alloway TP, Alloway RG. Investigating the predictive roles of working memory and IQ in academic attainment. Journal of Experimental Child Psychology. 2010; 106(1):20-9. [DOI:10.1016/j. jecp.2009.11.003] [PMID]

[6] Diamond A. Executive functions. Annual Review of Psychology. 2013; 64:135-68. [DOI:10.1146/annurev-psych-113011-143750] [PMID] [PMCID]

[7] Alizadeh H. [Neurocognitive executive functions and their relationship with developmental disorders (Persian)]. Advances in Cognitive Sciences. 2007; 8(4):57-70. http://icssjournal.ir/article1-269-en.html

[8] Brydges CR, Reid CL, Fox AM, Anderson M. A unitary executive function predicts intelligence in children. Intelligence. 2012; 40(5):458-69. [DOI:10.1016/j.intell.2012.05.006]

[9] Neuenschwander R, Röthlisberger M, Cimeli P, Roebers CM. How do different aspects of self-regulation predict successful adaptation to school? Journal of Experimental Child Psychology. 2012; 113(3):353-71. [DOI:10.1016/j.jecp.2012.07.004] [PMID]

[10] Murdock KW, Oddi KB, Bridgett DJ. Cognitive correlates of personality: Links between executive functioning and the big five personality traits. Journal of Individual Differences. 2013; 34(2):97-104. [DOI:10.1027/1614-0001/a000104]

[11] Razza RA, Blair C. Associations among false-belief understanding, executive function, and social competence: A longitudinal analysis. Journal of Applied Developmental Psychology. 2009; 30(3):332-43. [DOI:10.1016/j.appdev.2008.12.020] [PMID] [PMCID]

[12] Bridgett DJ, Valentino K, Hayden LC. The contribution of children's temperamental fear and effortful control to restraint and seclusion during inpatient treatment in a psychiatric hospital. Child Psychiatry \& Human Development. 2012; 43(6):821-36. [DOI:10.1007/s10578-012-0298-x] [PMID]

[13] Fuster JM. The prefrontal cortex: Anatomy, physiology, and neuropsychology of the frontal lobe. Philadelphia: Lippincott- Raven; 1997. https://books.google.com/ books?id=YupqAAAAMAAJ\&q

[14] Davidson MC, Amso D, Anderson LC, Diamond A. Development of cognitive control and executive functions from 4 to
13 years: Evidence from manipulations of memory, inhibition, and task switching. Neuropsychologia. 2006; 44(11):2037-78 [DOI:10.1016/j.neuropsychologia.2006.02.006] [PMID] [PMCID]

[15] Stuss DT, Benson DF. The frontal lobes. New York: Raven Press; 1986. https://books.google.com/ books?id=JO9qAAAAMAAJ\&q

[16] Diamond A, Barnett WS, Thomas J, Munro S. Preschool program improves cognitive control. Science. 2007; 318(5855):1387-8. [DOI:10.1126/science.1151148] [PMID] [PMCID]

[17] Carlson SM, White RE, Davis-Unger AC. Evidence for a relation between executive function and pretense representation in preschool children. Cognitive Development. 2014; 29:1-16. [DOI:10.1016/j.cogdev.2013.09.001] [PMID] [PMCID]

[18] Carlson SM, Zelazo PD, Faja S. Executive function. In: Zelazo PD, editor. The Oxford Handbook of Developmental Psychology. Vol. 1: Body and Mind. New York: Oxford University Press; 2013. p. 706-743. [DOI:10.1093/oxfordhb/9780199958450.013.0025]

[19] Müller U, Baker L, Yeung E. A developmental systems approach to executive function. In: Lerner RM, Benson JB, editor. Embodiment and Epigenesis: Theoretical and Methodological Issues in Understanding the Role of Biology within the Relational Developmental System Part B: Ontogenetic Dimensions. Advances in child development and behavior. Advances in Child Development and Behavior. Vol. 45. Waltham, MA: Academic Press; 2013. p. 39-66. [DOI:10.1016/B978-0-12-397946-9.00003-8] [PMID]

[20] Kamijo K, Hayashi Y, Sakai T, Yahiro T, Tanaka K, Nishihira $Y$. Acute effects of aerobic exercise on cognitive function in older adults. The Journals of Gerontology: Series B. 2009; 64B(3):356-63. [DOI:10.1093/geronb/gbp030] [PMID]

[21] Bahram A, Shafizadeh M. [Relationships between goal orientation and perceived ability with participation motivation in students (Persian)]. Journal of Movement Science \& Sports. 2006; 3(6):53-69. https://www.sid.ir/fa/journal/ViewPaper. aspx?id=62831

[22] Reeves L, Broeder CE, Kennedy-Honeycutt L, East C, Matney L. Relationship of fitness and gross motor skills for five- to six yr.-old children. Perceptual and Motor Skills. 1999; 89(3):739-47. [DOI:10.2466/pms.1999.89.3.739] [PMID]

[23] Cleland FE, Gallahue DL. Young children's divergent movement ability. Perceptual and Motor Skills. 1993; 77(2):535-44. [DOI:10.2466/pms.1993.77.2.535] [PMID]

[24] Piaget J, Inhelder B. La Psychologie De L'enfant. Paris: Presses Universitaires de France; 1966. https://www.persee.fr/doc/ pop_0032-4663_1967_num_22_5_11200

[25] Bushnell EW, Boudreau JP. Motor development and the mind: The potential role of motor abilities as a determinant of aspects of perceptual development. Child Development. 1993; 64(4):1005-21. [DOI:10.2307/1131323]

[26] Goldshtrom Y, Korman D Bendavid J. The effect of rhythmic exercise on cognition behavior of maltreated children: A pilot study. Journal of Bodywork and Movement Therapies. 2011; 15(3):326-34. [DOI:10.1016/j.jbmt.2010.06.006] [PMID]

[27] Brace JJ, Morton JB, Munakata Y. When actions speak louder than words: Improving children's flexibility in a card-sorting task. Psychological Science. 2006; 17(8):665-9. [DOI:10.1111/j.14679280.2006.01763.x] [PMID] 
[28] Castaño J. [Neurobiological bases of language and its disorders (Spanish)]. Revista de Neurologia. 2003; 36(8):781-5. [DOI:10.33588/rn.3608.2002206] [PMID]

[29] Schirmer CR, Fontoura DR, Nunes ML. [Language and learning disorders (Portuguese)]. Jornal de Pediatria. 2004; 80(2 Suppl):S95-103. [DOI:10.2223/1173]

[30] Blumstein SE, Cooper WE, Goodglass H, Statlender Sh, Gottlieb J. Production deficits in aphasia: A voice-onset time analysis. Brain and Language. 1980; 9(2):153-70. [DOI:10.1016/0093934X(80)90137-6]

[31] Selikowitz M. Dyslexia and other learning difficulties: The facts. Oxford: Oxford Medical Publications. 1997. https:// books.google.com/books?id=VcN6arZn6J4C\&dq

[32] Prizant BM, Audet LR, Burke GM, Hummel LJ, Maher SR, Theadore G. Communication disorders and emotional/behavioral disorders in children and adolescents. Journal of Speech and Hearing Disorders. 1990; 55(2):179-92. [DOI:10.1044/ jshd.5502.179] [PMID]

[33] Kuhn LJ, Willoughby MT, Wilbourn MP, Vernon-Feagans L, Blair CB, The Family Life Project Key Investigators. Early communicative gestures prospectively predict language development and executive function in early childhood. Child Development. 2014; 85(5):1898-914. [DOI:10.1111/cdev.12249] [PMID] [PMCID]

[34] Halse MK. Stability and predictors of change in executive functions from the age of 6 to 8 [MSc. thesis]. Trondheim: Norwegian University of Science and Technology; 2014. https://ntnuopen.ntnu.no/ntnu-xmlui/bitstream/handle/11250/271228/733750_FULLTEXT01.pdf

[35] Bernier A, Carlson SM, Whipple N. From external regulation to self-regulation: Early parenting precursors of young children's executive functioning. Child Development. 2010; 81(1):326-39. [DOI:10.1111/j.1467-8624.2009.01397.x] [PMID]

[36] Alizadeh H, Zahedipour M. [Executive functions in children with and without developmental coordination disorder (Persian)]. Advances in Cognitive Sciences. 2004; 6(3-4):49-56. http://icssjournal.ir/article-1-91-en.html

[37] Lambek R, Trillingsgaard A. Elaboration, validation and standardization of the Five to Fifteen (FTF) questionnaire in a Danish population sample. Research in Developmental Disabilities. 2015; 38:161-70. [DOI:10.1016/j.ridd.2014.12.018] [PMID]

[38] Abdolmohammadi K, Ghadiri Sourman Abadi F, Ebrahimzadeh Kh, Alizadeh A, Zarean M, Nourafkan F. [Psychometric properties of developmental -behavioral problems questionnaire (FTF 5-15) in Iranian children aged 5-15 years (Persian)]. Educational Measurement. 2020; 10(39):1-15. [DOI:10.22054/JEM.2020.44493.1939]

[39] Bishop DVM, Nation K, Patterson K. When words fail us: Insights into language processing from developmental and acquired disorders. Philosophical transactions of the Royal Society B Biological Sciences. 2014; 369(1634):20120403. [DOI:10.1098/rstb.2012.0403] [PMID] [PMCID]

[40] Gooch D, Hulme C, Nash HM, Snowling MJ. Comorbidities in preschool children at family risk of dyslexia. Journal of Child Psychology and Psychiatry. 2014; 55(3):237-46. [DOI:10.1111/ jcpp.12139] [PMID] [PMCID]
[41] Vocate DR. The theory of A. r. Luria: Functions of spoken language in the development of higher mental processes. Hillsdale, NJ: Lawrence Erlbaum Associates; 1987. https://books. google.com/books?id=h8J9Oq2Jh8C\&dq

[42] Luria AR. Language and cognition. Wertsch JV, editor. Hoboken: Wiley; 1982.

[43] Thomas JR, Moon DH. Measuring motor rhythmic ability in children. Research Quarterly. American Alliance for Health, Physical Education and Recreation. 1976; 47(1):20-32. [DOI:10.10 80/10671315.1976.10615336]

[44] Sutoo D, Akiyama K. Regulation of brain function by exercise. Neurobiology of Disease. 2003; 13(1):1-14. [DOI:10.1016/S09699961(03)00030-5]

[45] Golubović Š, Maksimović J, Golubović B, Glumbić N. Effects of exercise on physical fitness in children with intellectual disability. Research in Developmental Disabilities. 2012; 33(2):608-14 [DOI:10.1016/j.ridd.2011.11.003] [PMID]

[46] Diamond A. Close interrelation of motor development and cognitive development and of the cerebellum and prefrontal cortex. Child Development. 2000; 71(1):44-56. [DOI:10.1111/14678624.00117] [PMID]

[47] Davis EE, Pitchford NJ, Jaspan T, McArthur D, Walker D. Development of cognitive and motor function following cerebellar tumour injury sustained in early childhood. Cortex. 2010; 46(7):919-32. [DOI:10.1016/j.cortex.2009.10.001] [PMID] 
This Page Intentionally Left Blank 